\title{
Effects of Repetitive Transcranial Magnetic Stimulation on Motor Recovery in Lower Extremities of Subacute Stage Incomplete Spinal Cord Injury Patients: A Randomized Controlled Trial
}

\author{
Sang-Goo Ji ${ }^{1}$, Hyun-Gyu Cha ${ }^{2}$, and Myoung-Kwon Kim ${ }^{3 *}$ \\ ${ }^{1}$ Department of Physical Therapy, Eulji University Hospital, Daejeon 35233, Korea \\ ${ }^{2}$ Department of Physical Therapy, Kyungbuk College, Yeongju 36133, Korea \\ ${ }^{3}$ Department of Physical Therapy, College of Rehabilitation Sciences, Daegu University, Gyeongsan-si 38453, Korea
}

(Received 22 July 2015, Received in final form 18 August 2015, Accepted 18 August 2015)

\begin{abstract}
The aim of this study was to investigate whether repetitive transcranial magnetic stimulation (rTMS) can improve motor recovery in the lower extremities of the patients with subacute stage spinal cord injury (SCI). This study was conducted with 19 subjects diagnosed with paraplegia because of SCI. The experimental group included 10 subjects who underwent active rTMS, and the control group included 9 subjects who underwent sham rTMS. The SCI patients in the experimental group underwent conventional rehabilitation therapy, and active rTMS was applied daily to the hotspot of the lesional hemisphere. The SCI patients in the control group underwent sham rTMS and conventional rehabilitation therapy. The participants in both the groups received therapy five days per week for six weeks. Latency, amplitude, and velocity were assessed before and after the six-week therapy period. A significant difference in post-treatment gains for the latency and velocity was observed between the experimental and control groups $(p<0.05)$. However, no significant differences in the amplitude were observed between the two groups $(p>0.05)$. The results of this study indicate that $r$ TMS may be beneficial in improving motor recovery in the lower extremities of subacute stage SCI patients.
\end{abstract}

Keywords : repetitive transcranial magnetic stimulation, spinal cord injury, subacute stage

\section{Introduction}

Voluntary motion occurs when nerve impulses travel from the brain down the spinal cord and out to the body through the peripheral nerves. Sensory stimuli are carried from the peripheral nerves through the spinal cord to the brain. The full circuit enables tactile perception and coordinated movements. Nearly half of spinal cord injuries (SCI) are the result of motor vehicle crashes; other major causes include falls, violence, and sports accidents [1].

Damage to the spinal cord can result in both the loss of voluntary movement (paralysis) and sensation. When the spinal cord is damaged, communication between the brain and parts of the body that are innervated at or below the lesion is disrupted. The lesion may be complete (no nerve fibers are functioning below the level of injury) or incomplete (one or more nerve fibers are secure). Those

CThe Korean Magnetics Society. All rights reserved.

*Corresponding author: Tel: +82-53-850-4352

Fax:+82-53-850-4359, e-mail: skybird-98@hanmail.net who are injured at or below the thoracic level will have paraplegia, with function maintained in their upper extremities but some degree of impairment in the trunk and lower extremities. The American Spinal Injury Association Impairment Scale (AIS) was developed as a system for describing the severity of injury using letters that pertain to the extent of injury (usually A through D) and is widely used in the medical community [2]. AIS A injuries are complete, with no preservation of motor or sensory function below the neurological level of injury, including the sacral segments S4-S5. AIS B injuries are incomplete, with the preservation of sensory function, but not motor function below the neurological level of injury. AIS C and D classifications refer to incomplete injuries with the preservation of increasing degrees of motor function below the neurological level of injury. Injuries at the lumbar level often result in paraplegia. Persons with L1-L2 injury may be capable of standing and walking with a brace. However, this requires a significant amount of energy even for short time periods; a wheelchair will be needed for the mobility of any significant distance. 
Those with an L3-L4 injury may also be able to walk with orthotic devices [3].

Transcranial magnetic stimulation (TMS) might be an appropriate alternative treatment because of its simplicity, Single TMS pulses (rTMS) have been used as the noninvasive and painless method for stimulating the brain of intact conscious human subjects through the scalp [4]. Repeated applications of rTMS can sometimes elicit longlasting changes in the excitability of the corticospinal tract, M1, and spinal cord structures, significantly improving the sensory and motor function in the patients with motor disorders [5].

The applications of rTMS to the motor cortex at appropriate stimulation intensity can enable recording of motor evoked potentials (MEPs) from the muscles of the contralateral extremity. MEP amplitude can be affected by the type of cortical stimulator (high-voltage electrical or magnetoelectrical) and the stimulus intensity, as well as the activation of other muscles. It reflects not only the integrity of the corticospinal tract but also the excitability of the motor cortex and nerve roots and the conduction along the peripheral motor pathway to the muscles. The applications of rTMS in SCI have shown inconsistent results regarding the amelioration of pain. Belci et al. [6] examined somatomotor functional recovery in the patients with SCI and reported a short term reduction in cortical inhibition during the treatment with improved ASIA impairment scale measures of sensory and motor function and improved hand function that lasted into a recovery period. Nerve conduction is measured to assess the pathophysiological state of the nerves and is an essential tool for the study of various neuropathies. The clinical usefulness of nerve conduction studies (NCS) in the diagnosis of diffuse and focal neuropathies has been thoroughly validated [7].

In this study, we hypothesized that high frequency rTMS stimulation coupled with traditional physical therapy can improve motor recovery in the lower extremities SCI as compared to the same treatment with sham stimulation.

\section{Materials and Methods}

Thirty-nine patients with stroke were recruited from the neurological physical therapy clinic of the Faculty of Physical Therapy, Eulji University and were screened for this study from January 2015 to March 2015, after agreeing to participate in the study. All the patients were diagnosed with SCI, confirmed with computed tomography or magnetic resonance imaging. The patients who met the following criteria were enrolled: (1) incomplete SCI (AIS C or D) by trauma (traffic accident or fall down), (2) cervical or thoracic SCI, and (3) time lapse since SCI within six months.

A total of 19 SCI patients met the criteria. The Research Ethics Committee of Eulji University Hospital approved the study, and all the participants provided informed, written consent prior to the enrollment in the study.

When the initial assessment was completed, the subjects were randomly assigned to an experimental group ( $n$ $=10$ ) or a control group $(n=9)$. For randomization, sealed envelopes were prepared in advance and marked inside with A or B, indicating the experimental group or the control group. The randomization was performed by a third party who was unaware of the study content. The characteristics of the subjects and all the outcome measures before and after the treatment were assessed by Physician 1, who was blinded to the treatment allocations. The rTMS and sham treatments were administered in a closed room by Physician 2, who was not involved in the assessment of the subjects. Both physicians were instructed not to communicate with the subjects about the possible goals or the rationale of either treatment.

The subjects in the experimental group received rTMS and conventional rehabilitation therapy for a total of 50 min (rTMS: $20 \mathrm{~min}$; conventional rehabilitation therapy: $30 \mathrm{~min}$ ) per day, with a $10 \mathrm{~min}$ rest period halfway through the session. Magstim Rapid2 (Magstim Co., Ltd, Wales, United Kingdom) was used for rTMS, using a figure-ofeight coil with a diameter of $80 \mathrm{~mm}$.

The subjects in the experimental group received training five days per week for four weeks. Conventional rehabilitation therapy, consisting of neurodevelopmental facilitation techniques, was administered by the therapists blinded to the study protocol and subjects' assignment to groups. The objectives of conventional rehabilitation therapy were to improve patients' functional abilities including transfer, ambulation, and balance to help patients achieve earlier and/or greater independence in daily-life activities. The subjects in the control group received sham therapy and conventional rehabilitation therapy for a total of 50 min (sham rTMS: 20 min, conventional rehabilitation therapy: $30 \mathrm{~min}$ ) per day on the same day.

The initial current induced by the biphasic pulse of the magnetic stimulator would have flowed posterolaterally [8]. The junction of the coil was placed over the lowest threshold spot for eliciting a MEP contralaterally in the abductor hallucis muscles. The muscle with the lowest rTMS threshold, left or right side, was selected as the reference muscle for applications of rTMS and determined as the hemisphere to be stimulated. The subjects were seated with their lower limbs relaxed, and rTMS was delivered at $10 \mathrm{~Hz}$ as $2 \mathrm{~s}$ trains separated by $8 \mathrm{~s}$ for 


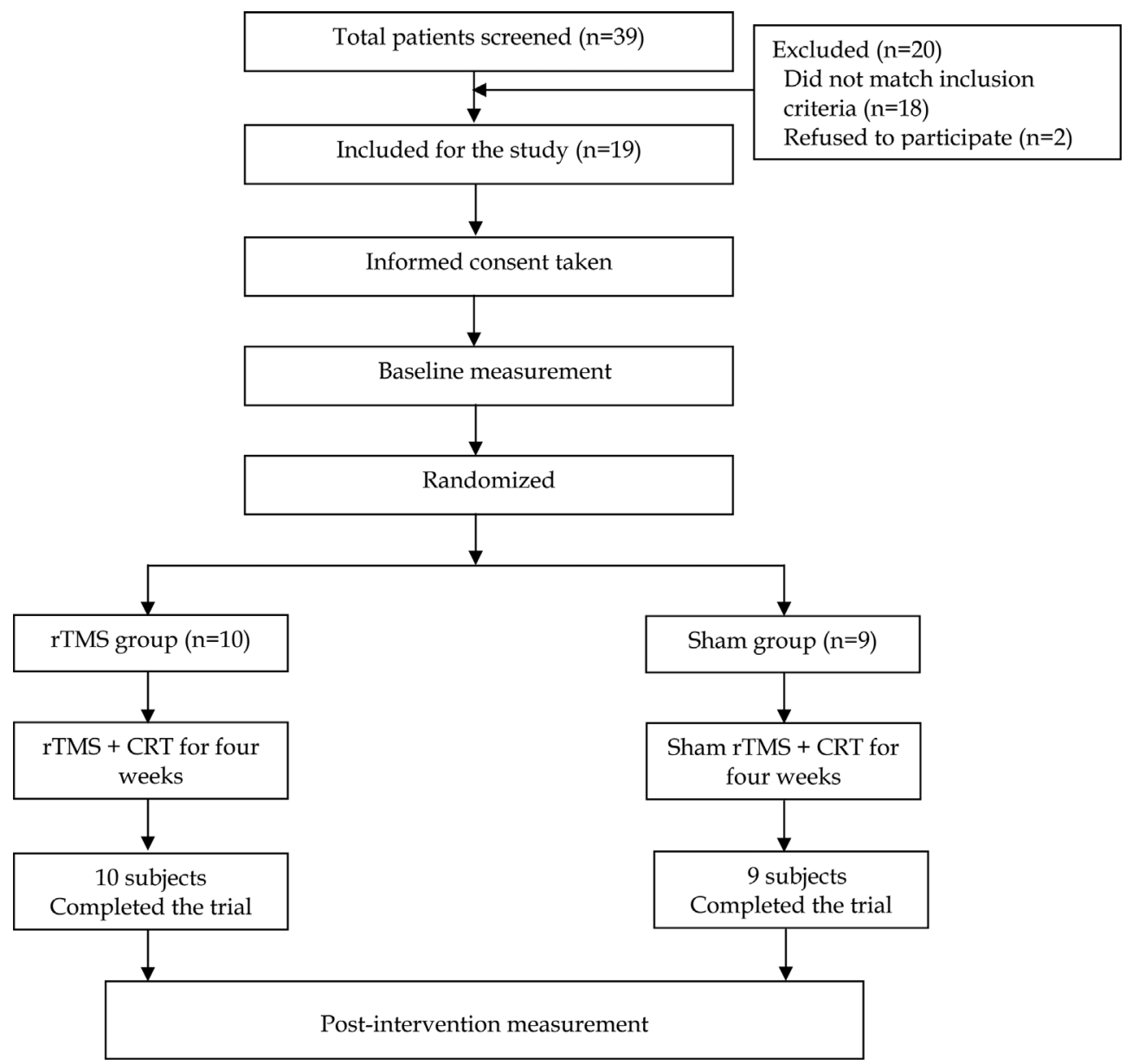

Fig. 1. Study flowchart. CRT: comprehensive rehabilitation therapy.

20 min. Stimulation was applied at $80 \%$ of the active motor threshold for eliciting an MEP during a weak $(\sim 10 \%)$ voluntary contraction. Those in the control group were led to hear sounds using a sham stimulator coil without knowing that the sounds were because of the sham stimulation [9].

All the participants were blinded to the rTMS condition, and none of them had any experience with rTMS before precipitating in he study. The EMG/EP system was used for the measurement of Motor nerve conduction velocity (MNCV). Stimulus was applied to the common peroneal nerve in the fibular head, and MNCV was recorded in extensor digiti brevis. Stimulus frequency was set in the range $\sim 2 \mathrm{~Hz}-10 \mathrm{kHz}$ with the intensity in the range $\sim 40-50 \mathrm{~mA}$, and the time was $0.5 \mathrm{~s}$ [10].

The data are given as mean $\pm \mathrm{SD}$ values. All the variables were tested for normality using the KolmogorovSmirnov test and did not show a normal distribution. The Wilcoxon signed-rank test was used for comparisons of the variables before and after training within each group, and the Mann-Whitney U test was used for comparisons of pre- and post-test differences in the variables between the experimental and control groups. Analyses were performed using PAWS, version 18.0 (SPSS Inc., Chicago, IL, USA), and $p<0.05$ was regarded as the criterion for statistical significance.

\section{Results}

A summary of the clinical and demographic features of the sample $(n=19)$ is listed in Table 1 . The values of the MEP and NCV of both the groups are listed in Table 2. Significant differences in the post-test results for latency and velocity were observed between the two groups $(\mathrm{p}<$ 0.05 ). In both the groups, significant differences were found in the pre- and post-test results of all the variables $(\mathrm{p}<0.05)$.

\section{Discussion}

This study was conducted to investigate the effects of active rTMS applied for the improvement of motor 
Table 1. Subject demographics

\begin{tabular}{|c|c|c|c|c|c|c|}
\hline rTMS & Gender & Age & $\begin{array}{l}\text { Time since } \\
\text { injury } \\
\text { (months) }\end{array}$ & AIS & $\begin{array}{c}\text { Level of } \\
\text { injury }\end{array}$ & Cause \\
\hline Active & $\mathrm{M}$ & 19 & 5 & D & $\mathrm{C} 4$ & $\mathrm{TA}$ \\
\hline Active & M & 29 & 6 & $\mathrm{D}$ & C6 & $\mathrm{F}$ \\
\hline Active & $\mathrm{F}$ & 38 & 4 & $\mathrm{C}$ & $\mathrm{T} 2$ & F \\
\hline Active & M & 35 & 3 & D & C7 & TA \\
\hline Active & M & 49 & 2 & $\mathrm{C}$ & $\mathrm{T} 7$ & TA \\
\hline Active & $\mathrm{F}$ & 42 & 5 & D & $\mathrm{T} 4$ & $\mathrm{~F}$ \\
\hline Active & $\mathrm{F}$ & 37 & 6 & $\mathrm{D}$ & T9 & TA \\
\hline Active & M & 35 & 1 & $\mathrm{D}$ & C5 & $\mathrm{F}$ \\
\hline Active & M & 56 & 3 & $\mathrm{C}$ & $\mathrm{T} 5$ & $\mathrm{TA}$ \\
\hline Active & $\mathrm{M}$ & 59 & 5 & $\mathrm{D}$ & C5 & $\mathrm{TA}$ \\
\hline Sham & M & 21 & 3 & D & $\mathrm{C} 7$ & $\mathrm{~F}$ \\
\hline Sham & M & 57 & 3 & $\mathrm{D}$ & C6 & TA \\
\hline Sham & $\mathrm{F}$ & 52 & 2 & $\mathrm{C}$ & $\mathrm{T} 8$ & $\mathrm{~F}$ \\
\hline Sham & $\mathrm{F}$ & 43 & 5 & $\mathrm{D}$ & $\mathrm{T} 7$ & TA \\
\hline Sham & M & 48 & 6 & $\mathrm{D}$ & $\mathrm{T} 4$ & TA \\
\hline Sham & $\mathrm{F}$ & 39 & 2 & $\mathrm{D}$ & C6 & $\mathrm{F}$ \\
\hline Sham & M & 47 & 2 & $\mathrm{C}$ & C5 & TA \\
\hline Sham & M & 32 & 4 & D & T6 & $\mathrm{TA}$ \\
\hline Sham & M & 26 & 6 & $\mathrm{D}$ & $\mathrm{T} 8$ & $\mathrm{TA}$ \\
\hline
\end{tabular}

AIS: American Spinal Cord Injury Association Impairment Scale, C: cervical level, T: thoracic level, TA: traffic accident, F: fall down

Table 2. Comparison of MEP and NCS between groups $(\mathrm{N}=$ 19)

\begin{tabular}{lcccc}
\hline \hline & & EG $(\mathrm{n}=10)$ & $\mathrm{CG}(\mathrm{n}=9)$ & $\mathrm{Z}$ \\
\hline \multirow{2}{*}{ Latency $(\mathrm{ms})^{\dagger}$} & Pre & $34.21 \pm 5.13^{\mathrm{a}}$ & $35.36 \pm 4.75$ & -0.41 \\
& Post & $29.44 \pm 3.64^{*}$ & $36.63 \pm 5.12^{*}$ & -2.65 \\
\hline \multirow{2}{*}{ Amplitude $(\mathrm{mV})$} & Pre & $0.25 \pm 0.14$ & $0.31 \pm 0.15$ & -0.87 \\
& Post & $0.51 \pm 0.12^{*}$ & $0.41 \pm 0.19^{*}$ & -1.17 \\
\hline \multirow{2}{*}{$\mathrm{MNCV}(\mathrm{m} / \mathrm{s})^{\dagger}$} & Pre & $43.05 \pm 5.13$ & $42.51 \pm 3.30$ & -0.65 \\
& Post & $49.53 \pm 5.14^{*}$ & $44.23 \pm 4.02^{*}$ & -2.24 \\
\hline
\end{tabular}

${ }^{\mathrm{a}}$ mean $\pm \mathrm{SD}$

${ }^{*} \mathrm{p}<0.05$; significant difference compared with the pretest

$\uparrow p<0.05$; significant difference compared with the control group EG, rTMS, and conventional physical therapy groups; CG: Sham rTMS and conventional physical therapy groups

MNCV: Motor nerve conduction velocity

recovery in the lower extremities in the patients with subacute stage SCI. The results of this study show that latency and $\mathrm{MNCV}$ were more enhanced in the experimental group than the control group after the intervention. Active rTMS was more effective than sham rTMS in improving motor recovery.

According to another study, rTMS could reduce motor improvement in SCI. It is possible that motor score improvement and amelioration of spasticity with rTMS in SCI can be induced through the enhancement of descend- ing corticospinal projection and reduction in corticospinal inhibition [11].

Using a sample of 17 SCI AIS D level patients, Benito et al. [12] applied $20 \mathrm{~Hz}$ high-frequency rTMS to an experimental group consisting of seven patients and sham rTMS to the control group consisting of ten patients, for 15 days each and reported that the experimental group showed significant improvement in the modified Ashworth scale (MAS), $10 \mathrm{~m}$ walking test (MWT), cadence, step length, and timed up and go test (TUG) compared to the control group.

Belci et al. [6] reported the improvement in the motor score and upper extremity function in four patients with chronic incomplete cervical SCI after the application of rTMS over the motor cortex for five days.

The MEP elicited by TMS represents a highly accurate diagnostic test with a very high sensitivity value in SCI [13]. Rossini [14] argued that the amplitude increases and the latency decreases as descending tract excitability increases. In this study, as latency decreased significantly in the experimental group compared to the control group, the application of rTMS was found to be effective in excitability recovery. Regarding the NCV test in upper motor neuron diseases, in the NCV test of paralyzed and affected side peroneal nerves, Martinez et al. [15] reported that statistical difference was observed only in the paralyzed side peroneal nerve. This study confirmed the motor function recovery effects of the lower limb in the experimental group compared to the control group with increased NCV.

These results support the perceived benefit of rTMS in augmenting motor recovery of the subacute stage patients within the first six months of SCI. The current study has some limitations. First, the small sample size may have affected certain variables, thus affecting the results. Therefore, these results cannot be generalized to all the SCI patients. Second, the absence of follow-up after the end of the active rTMS does not allow the determination of the durability of the effect of this intervention. Further studies, including a long-term follow-up assessment, are needed to evaluate the long-term benefits of rTMS.

\section{References}

[1] A. Jardin, G. Wagner, S. Khoury, F. Guiliano, H. Padman, and N. Rosen, J. Spinal Cord. Med. 23, 153 (2000).

[2] R. J. Marino, T. Barros, F. Biering, S. P. Burns, W. H. Donovan, and D. E. Graves, J. Spinal Cord. Med. 26, 50 (2003).

[3] N. M. Crewe and J. S. Krause, Rehabil Psycho. 35, 205 (1990). 
[4] A. T. Baker, R. Jalinous, and I. L. Freeston, Lancet 1, 1106 (1985).

[5] M. C. Ridding and J. C. Rothwell, Nat. Rev. Neurosci. 8, 559 (2007).

[6] M. Belci, M. Catley, M. Husain, H. L. Frankel, and N. J. Davey, Spinal Cord. 42, 417 (2004).

[7] W. W. Campbell, L. C. Ward, and T. R. Swift, Muscle Nerve 3, 436 (1981).

[8] D. Balslev, W. Braet, C. McAllister, and R. C. Miall, J. Neurosci Methods 162, 309 (2007).

[9] S. G. Ji and M. K. Kim, J. Magn. 20, 129 (2015).

[10] M. M. LaBan, D. Petty, A. M. Hauser, and R. S. Taylor, Arch. Phys. Med. Rehabil. 69, 358 (1988).
[11] H. Kumru, N. Murillo, and J. V. Samso, Neurorehabil Neural Repair 24, 435 (2010).

[12] J. Benito, H. Kumru, N. Murillo, U. Costa, J. Medina, J. M. Tormos, P. L. Alvaro, and J. Vidal, Top Spinal Cord. Inj. Rehabil. 18, 106 (2012).

[13] V. Lazzaro, A. Oliviero, P. Profice, L. Ferrara, E. Saturno, F. Pilato, and P. Tonali, Clin Neurophysiol 110, 1297 (1999).

[14] P. M. Rossini, M. Carmia, and F. Zarola, Neurosurg 20, 183 (1987).

[15] A. Martinez, Electromyogr Clin Neurophysiol 23, 139 (1983). 